\title{
Optimal subpixel interpolation in particle image velocimetry
}

\author{
T. Roesgen
}

Abstract It is shown that among the different techniques
for particle image velocimetry subpixel interpolation, only
the "sinc"-kernel creates an optimal result in that it
completely suppresses spurious spectral sidelobes. An
efficient method is introduced for the computation of the
subpixel-accurate correlation peak position without any
systematic errors. A connection is made with the kernel-
dependent observation of the peak-locking phenomenon.

1

\section{Introduction}

The accuracy of the velocity estimate in particle image velocimetry (PIV) depends on the successful control or elimination of a number of error sources. Typical areas of concern are the under-resolved optical sampling of the particle images, the presence of velocity gradients in the subwindow being analyzed, and inaccurate interpolation of the correlation peak at the subpixel level. Here, a procedure will be introduced that eliminates errors associated with the last problem area.

\section{2}

\section{Interpolation and convolution}

The interpolation procedure of a sampled data sequence can be split up into two separate steps, the interleaving of the original data with zero values ("zero padding") and the subsequent convolution with a suitable kernel. The Fourier transform of the zero-padded data replicates the original data spectrum at integer multiples of the sampling frequency (Alexander and $\mathrm{Ng} \mathrm{1991).} \mathrm{The} \mathrm{interpolating}$ convolution kernel is multiplied onto this extended spectrum and performs a low-pass filtering operation. Care has to be taken in the choice of the kernel to avoid errors caused by the inclusion of the extraneous high-frequency information. Figure 1 demonstrates this procedure with a one-dimensional example. A Gaussian pulse is to be interpolated to increase the resolution by a factor of five. The original data (sampled with a rate $\Delta$ ) is zero-padded with a sequence of four zeros following each sample. The

Received: 15 May 2002 / Accepted: 18 March 2003

Published online: 26 July 2003

(C) Springer-Verlag 2003

T. Roesgen

Institute of Fluid Dynamics, Swiss Federal Institute of Technology,

ETH Zentrum, Sonneggstrasse 3, 8092 Zurich, Switzerland

E-mail: roesgen@ifd.mavt.ethz.ch resulting spectrum is shown in Fig. 1a, with the fivefold replication of the original Fourier spectrum. In addition, the spectral response of two simple interpolation kernels (nearest neighbor, linear) is depicted whose (continuous) spatial profile is given in Eq. (1), based on a sampling interval $\Delta$,

$$
k_{\mathrm{nn}}(x)=\left\{\begin{array}{ll}
1 & |x / \Delta| \leqslant 0.5 \\
0 & |x / \Delta|>0.5
\end{array} k_{\operatorname{lin}}(x)=\left\{\begin{array}{cc}
1-|x / \Delta| & |x / \Delta| \leqslant 1 \\
0 & |x / \Delta|>1
\end{array}\right.\right.
$$

Nearest-neighbor interpolation is performed with a top hat kernel, resulting in a "sinc" function in the Fourier domain. Similarly, linear interpolation implies a triangular kernel and a "sinc" spectrum. Neither kernel completely suppresses the high-frequency replicas of the original spectrum and thus will introduce interpolation errors due to "spectral leakage". Another source of error is the low pass filtering effect introduced in the pass-band $|\pi / \Delta|<1$.

In Fig. 1b, three other filter kernels are introduced which perform better, since their Fourier coefficients decay rapidly in the stop-band. The " $\mathrm{M}^{\prime} 4$ " kernel

$k_{M^{\prime} 4}(x)=\left\{\begin{array}{cc}1-\frac{5}{2}(x / \Delta)^{2}+\frac{3}{2}|x / \Delta|^{3} & |x / \Delta| \leqslant 1 \\ \frac{1}{2}(2-|x / \Delta|)^{2}(1-|x / \Delta|) & 1 \leqslant|x / \Delta| \leqslant 2 \\ 0 & |x / \Delta|>2\end{array}\right.$

is often used in numerical simulations involving particles, where irregular data have to be resampled onto a regular grid. This kernel is (nearly) optimal in that it has a cutoff at half the sampling frequency, almost no sidelobes and a finite support in the data domain.

In the same spirit, Lourenco and Krothapalli (1995) introduced a truncated "sinc"-interpolation ("Whittaker") kernel that creates a similar, albeit oscillatory, spectral response:

$k_{\text {Whittaker }}(x)=\left\{\begin{array}{cl}\sin (\pi x / \Delta) /(\pi x / \Delta) & |x / \Delta| \leqslant 2 \\ 0 & |x / \Delta|>2\end{array}\right.$

If there is no constraint on the spatial (support) size, the optimum interpolator has a "sinc" shape in space and a "top hat" spectrum with the cutoff at half the sampling frequency:

$k_{\text {optimal }}(x)=\sin (\pi x / \Delta) /(\pi x / \Delta) \quad(-\infty<x<\infty)$ 

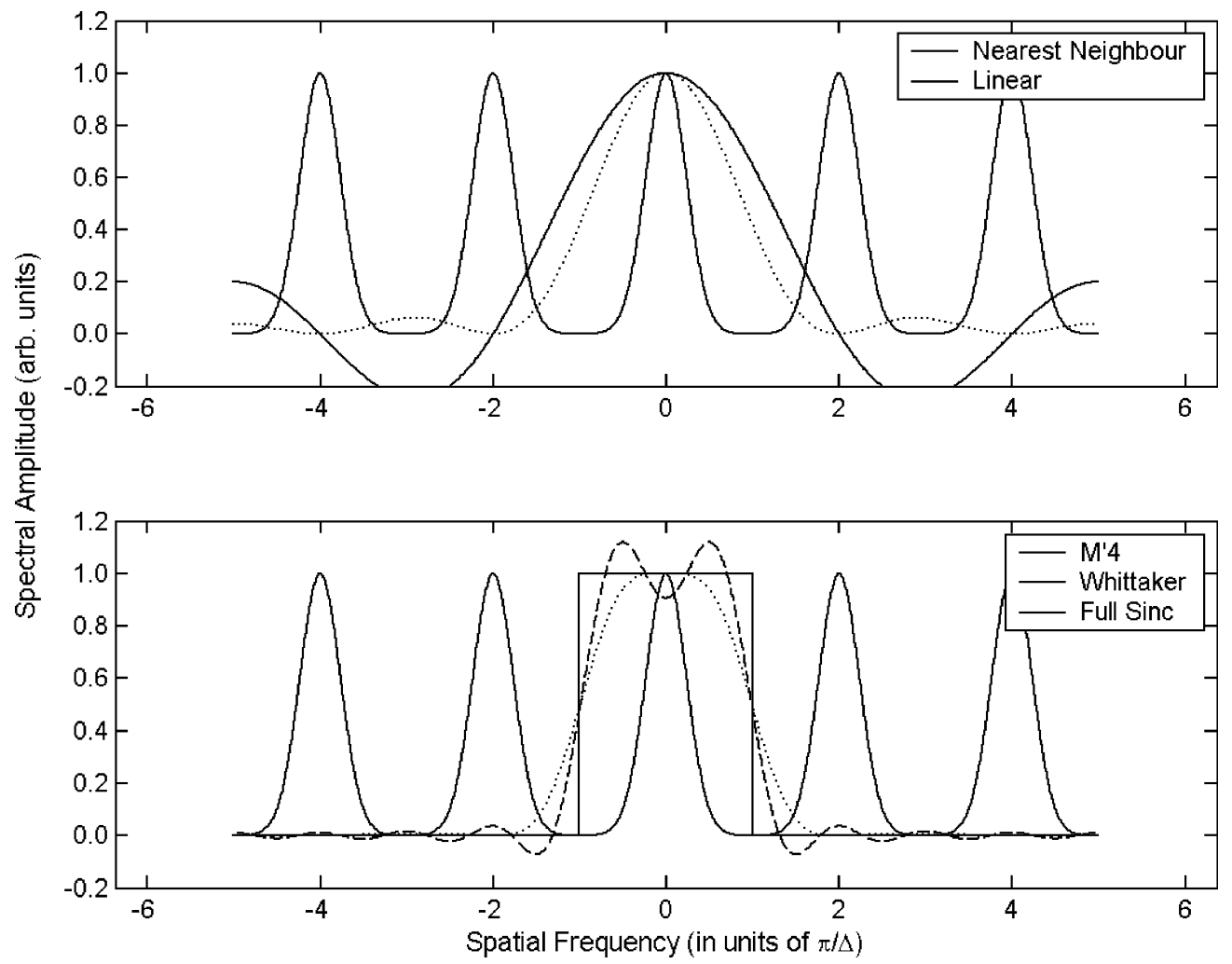

Fig. 1. Spectral shape of different subpixel interpolation kernels

Looking at the effect of this kernel on the zero-padded spectrum, it is clear that it completely eliminates the influence of the unwanted higher spectral replicas while it does not introduce any additional weighting/distortion inside the pass band. The full "sinc" kernel is thus proposed as the optimal function for use in PIV subpixel interpolation.

In many PIV interpolation schemes, another parametric approach is followed in that the local peak shape is approximated by a Gaussian or quadratic function. While no fixed, explicit kernel can be formulated beforehand in these cases, the operation can still be understood as a convolution: a Dirac delta function at the exact position of the correlation peak is convolved with the parametric

shape function. The impact of these heuristic interpolation schemes will be included in the discussion of the observed peak locking errors (Sect. 4).

\section{3}

\section{Fast peak search}

Looking for an implementation of the "sinc"-interpolation scheme, a first choice might be a direct summation formula

$c(x, y)=\sum_{m=0}^{M-1} \sum_{n=0}^{N-1} c(m \Delta, n \Delta) \operatorname{sinc}(x-m \Delta) \operatorname{sinc}(y-n \Delta)$

where $c(x, y)$ is the interpolated result and $c(m \Delta, n \Delta)$ the original, sampled data. This formula, however, will not work very well because of the finite-size data set. Problems become visible especially at the edge of the original data interval, where the interpolation becomes increasingly asymmetric and inaccurate because of the missing data points. In applying Eq. (5), one implicitly multiplies the discrete data set with a finite-width windowing function. This corresponds to a convolution of the spectrum with a blurring kernel which will lead again to interpolation errors due to spectral leakage, as discussed above.

The clean way to avoid this problem is to assume the original data set is continued periodically beyond the primary interval. Summing over an extended area of support leads to additional computational complexity which can be avoided by working directly in the Fourier domain. The transform coefficients of the correlation map are often available as part of the FFT-based computation of the cross-correlation. They are defined as

$\hat{C}(k, l)=\Delta^{2} \sum_{n=0}^{N-1} \sum_{m=0}^{M-1} c(m \Delta, n \Delta) \exp \left[-j 2 \pi\left(\frac{m k}{M}+\frac{n l}{N}\right)\right]$

This definition implicitly assumes a periodic data set, and no explicit extension is necessary. At the unknown position $\left(x_{0}, y_{0}\right)$ of the true correlation peak, one may write for the peak value with the inverse Fourier transform

$c\left(x_{0}, y_{0}\right)=\frac{1}{M N \Delta^{2}} \sum_{l=0}^{N-1} \sum_{k=0}^{M-1} \hat{C}(k, l) \exp \left[j 2 \pi\left(\frac{k x_{0}}{M \Delta}+\frac{l y_{0}}{N \Delta}\right)\right]$

The direct search for the peak position can be replaced by a search for a set of phase factors

$$
\begin{array}{r}
\phi_{k l}\left(x_{0}, y_{0}\right)=\exp \left[j 2 \pi\left(\frac{k x_{0}}{M \Delta}+\frac{l y_{0}}{N \Delta}\right)\right] \\
(k=0 \ldots M-1, l=0 \ldots N-1)
\end{array}
$$


Correlation Map
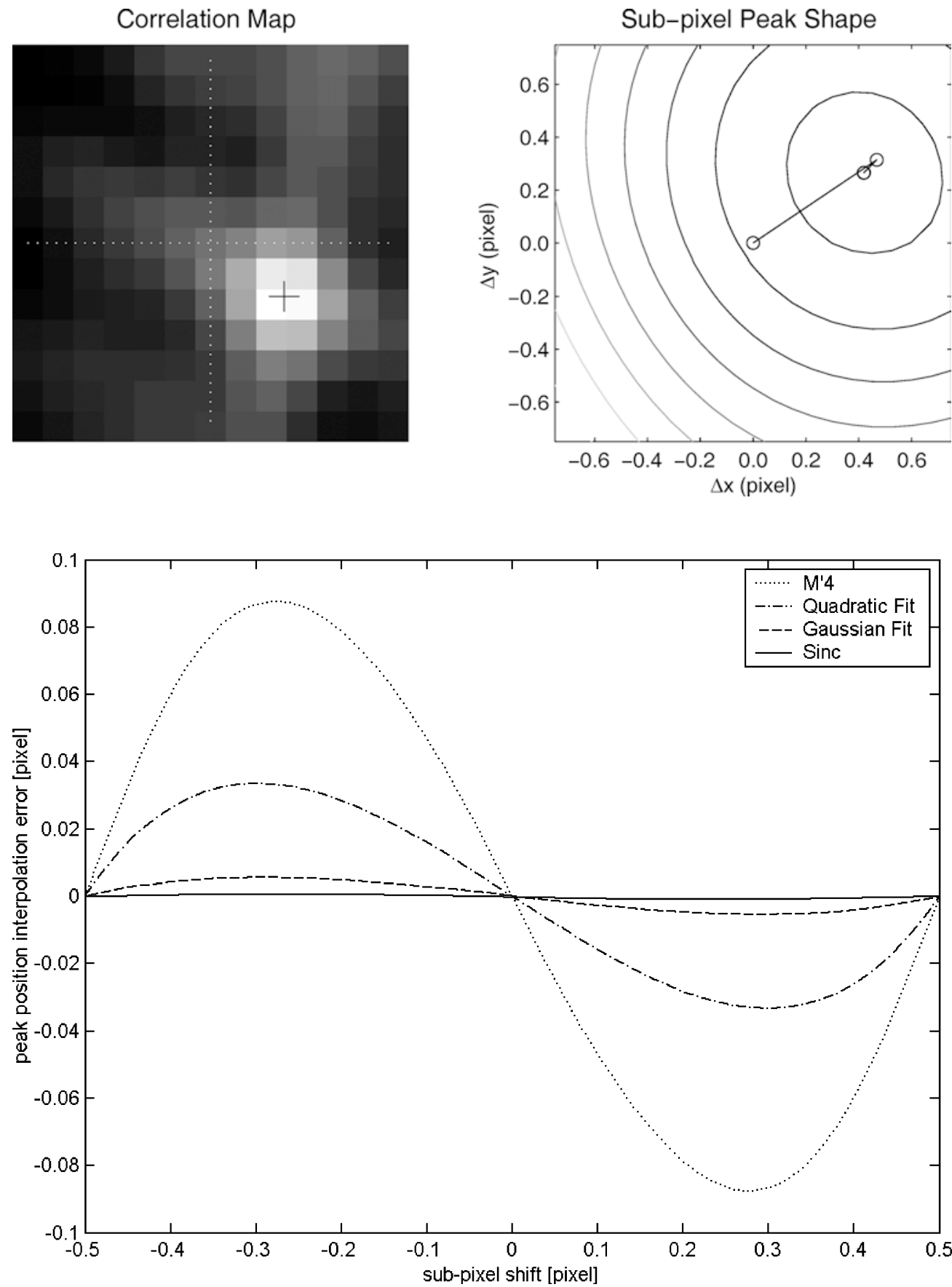

Fig. 2. Correlation map and detailed shape of the correlation peak, interpolated with the optimal "sinc" function

Fig. 3. Subpixel interpolation error for different interpolation schemes

which maximizes the sum in Eq. (7). An iterative scheme can be used to solve this nonlinear equation for $\left(x_{0}, y_{0}\right)$. Following Newton's method, for example, successive updates to the solution can be found as

$\underline{x}_{0}^{(n+1)}=\underline{x}_{0}^{(n)}+\Delta \underline{x}^{(n)}=\underline{x}_{0}^{(n)}-\left[\underline{\nabla} \underline{\nabla} c\left(\underline{x}_{0}^{(n)}\right)\right]^{-1} \underline{\nabla} c\left(\underline{x}_{0}^{(n)}\right)$

The gradient $\underline{\nabla} c\left(x_{0}, y_{0}\right)$ and the Hessian $\underline{\nabla} \underline{\nabla} c\left(\underline{x}_{0}^{(n)}\right)$ can be approximated by finite differences or computed explicitly from Eq. (7) to accelerate the convergence. A good starting estimate is provided by the integer shift coordinates as available from the non-interpolated crosscorrelation result. Using simulated data (see below), the iteration (9) converges in about five steps in that it reduces the length of the update vector $\left\|\underline{\Delta x}^{(n)}\right\|$ to less than $10^{-6}$ pixel.

It is important to remember that in the formulation of Eq. (7), no approximations are made and that an ideal interpolation is performed on a periodically continued data set. The converged estimate for the peak position contains no systematic errors caused by spectral leakage or low-pass filtering.

The shape of function $c()$ near its maximum, that is a typical correlation peak, is shown in Fig. 2. The contour plot expands the area around the map's peak value. Synthetic PIV data were used as provided by the Visualization Society of Japan (http://www.vsj.or.jp/piv/). A FFT-based cross-correlation algorithm was employed resulting in a 


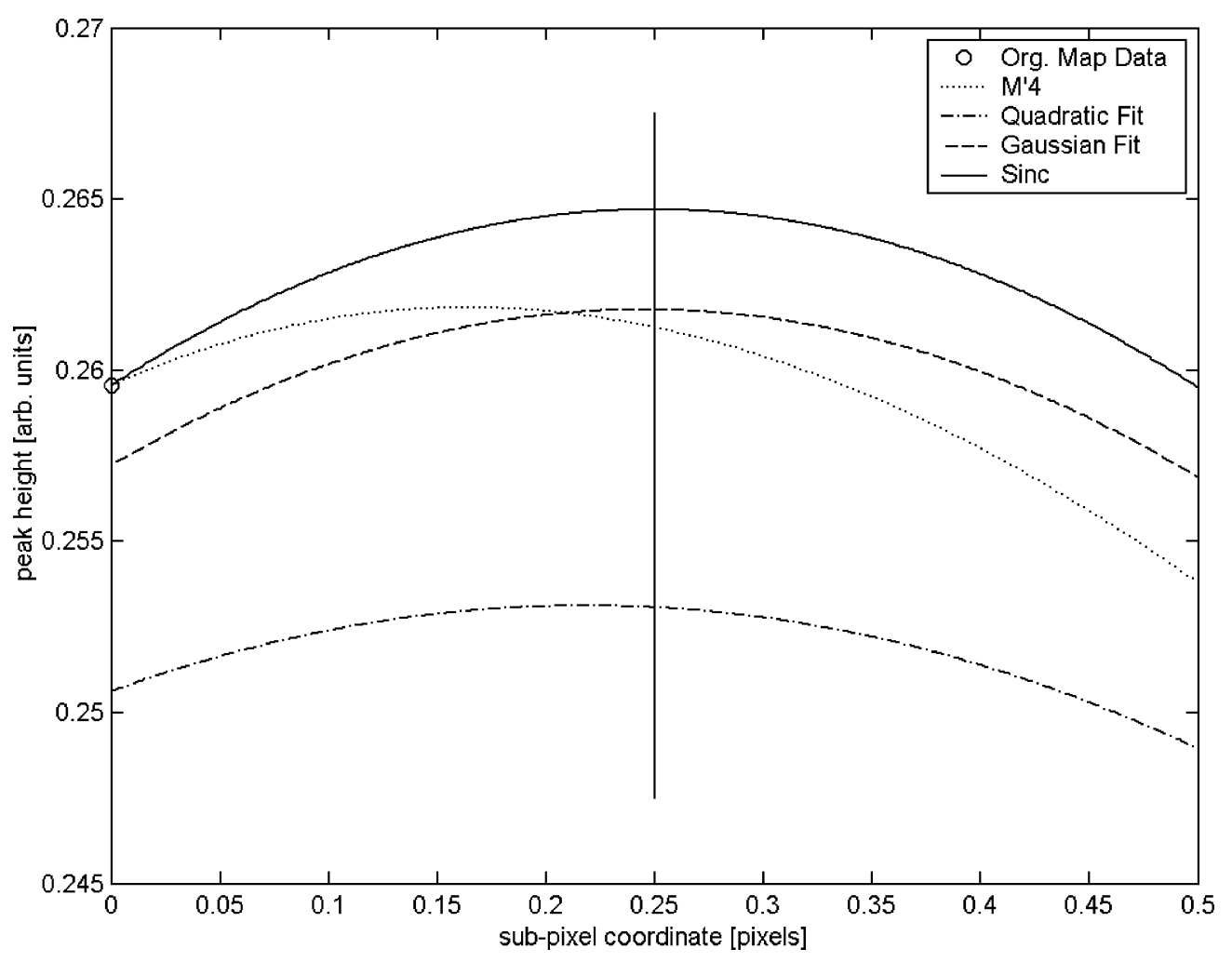

Fig. 4. Detailed correlation peak shape using different interpolators
$13 \times 13$-pixel correlation map. One recognizes the fairly regular, convex topology of the interpolated peak, justifying the use of the simple nonlinear maximizer. The trajectory plotted in Fig. 2 shows the descent path (three steps) of the iterations in Newton's algorithm.

The presented algorithm is in some respect similar to the one described by Sjödahl (1994). The direct implementation in Fourier space, however, greatly simplifies the processing.

\section{4}

\section{Peak locking interpreted as interpolation error}

In many publications dealing with PIV subpixel interpolation, a so-called "peak-locking" effect is discussed, which is the tendency of peak position estimates to cluster around integer values (Keane and Adrian 1992; Westerweel 1998; Fincham and Delerce 2000; Forliti et al. 2000; Nogueira et al. 2001; Gui and Wereley 2002; Scarano (2002). The empirical finding is that a Gaussian peak fit performs better than linear or quadratic interpolation (see e.g. Willert and Gharib 1991).

This observation is readily explained within the framework of Sect. 2: The Gaussian filter kernel suppresses the spurious spectral components quite efficiently. Still, the implicit "sinc"-interpolator does perform inherently better, since it does not attenuate the pass-band data.

Figure 3 presents the results of performing the subpixel interpolation with different interpolation kernels. The PIV data set is again based on a simulated picture from the source mentioned above $(256 \times 256$-pixel resolution). The second image in the PIV pair is created by a controlled global horizontal image shift of the first image, using a "sinc"-interpolator on the whole image. It is important to keep in mind that the subpixel interpolation operates on sub-images $(32 \times 32$ pixels $)$ so that the recovery of the precise local image shifts is non-trivial. The correlation maps ( $13 \times 13$ pixels) of 676 interrogations were averaged to provide a good estimate of the shifted correlation peaks (Meinhart et al. 2000). The peak position was then estimated using parametric peak fits using local $(3 \times 3)$ quadratic and Gaussian functions, as well as the $\mathrm{M}^{\prime} 4$ and full "sinc" interpolation kernels. The procedure was repeated for horizontal shifts in the range $-0.5<\delta<0.5$ pixels (steps size 0.02 pixel). The deviation from the expected linear subpixel displacement is plotted and one recognizes the minimal error signature of the "sinc" interpolator compared to the other schemes.

The impact of the interpolator choice is further visualized in Fig. 4, where the neighborhood around the correlation peak (true displacement 0.25 pixels, 1-D projection along the direction of the pattern shift) is shown. Again, the Gaussian fit provides the best results among the established interpolators, only to be outperformed by the "sinc" interpolator. Note also that only the two proper interpolation schemes generate curves which pass through the original correlation map data point(s). The parametric curve fits do not possess this property.

\section{5}

\section{Summary}

A fast, ideal "sinc" interpolator was introduced to compute a bias-free subpixel estimate of the correlation peak position in PIV. Using a direct computational scheme in the Fourier domain, problems with the direct interpolation of a finite data set are avoided. The claim is made that this "sinc"-interpolation also suppresses the interpolationrelated part of the peak-locking error in the most efficient manner. Any remaining systematic estimation errors will 
be associated with the other causes mentioned in the introduction: under-resolved particle images and unresolved spatial velocity gradients in the subwindows. The first error source cannot be eliminated a posteriori, whereas the second requires a different kind of pattern matching such as continuous coordinate warping.

\section{References}

Alexander BF, Ng KC (1991) Elimination of systematic error in subpixel accuracy centroid estimation. Opt. Eng 30:1320-1331

Fincham A, Delerce G (2000) Advanced optimization of correlation imaging velocimetry algorithms. Exp Fluids 29:S13-S22

Forliti DJ, Strykowski PJ, Debatin K (2000) Bias and precision errors of digital particle image velocimetry. Exp Fluids 28:436-447

Gui L, Wereley ST (2002) A correlation-based continuous windowshift technique to reduce the peak locking effect in digital PIV image evaluation. Exp Fluids 32:506-517
Keane RD, Adrian RJ (1992) Theory of cross correlation analysis of PIV images. J Appl Sci Res 49:191-215

Lourenco I, Krothapalli A (1995) On the accuracy of velocity and vorticity measurements with PIV. Exp Fluids 18:421-428

Meinhart CD, Wereley ST, Santiago JG (2000) A PIV algorithm for estimating time-averaged velocity fields. J Fluids Eng 122:285-289

Nogueira J, Lecuona A, Rodriguez PA (2001) Identification of a new source of peak locking, analysis and its removal in conventional and super-resolution PIV techniques. Exp Fluids 30:309-316

Scarano F (2002) Iterative image deformation methods in PIV. Meas Sci Technol 13:R1-R19

Sjödahl M (1994) Electronic speckle photography: increased accuracy by nonintegral pixel shifting. Appl Optics 33(28):6667-66673

Westerweel J (1998) Effect of sensor geometry on the performance of PIV interrogation. In: Adrian RJ, Durao DFG, Durst F, Heitor MV, Maeda M,Whitelaw JH (eds) Laser techniques applied to fluid mechanics. Springer, Berlin Heidelberg New York

Willert CE, Gharib M (1991) Digital particle image velocimetry. Exp Fluids 10:181-193 